\title{
Médiévales
}

Langues, Textes, Histoire

65 | automne 2013

Le couple dans le monde franc

\section{Du couple et des couples à l'époque carolingienne}

couple and couples from the Carolingian Times

\section{Janet Nelson}

Traducteur : Sylvie Joye

\section{OpenEdition}

\section{Journals}

Édition électronique

URL : https://journals.openedition.org/medievales/7075

DOI : 10.4000/medievales. 7075

ISSN : $1777-5892$

Éditeur

Presses universitaires de Vincennes

Édition imprimée

Date de publication : 1 décembre 2013

Pagination : 19-31

ISBN : 978-2-84292-396-9

ISSN : 0751-2708

\section{Référence électronique}

Janet Nelson, « Du couple et des couples à l'époque carolingienne », Médiévales [En ligne], 65।

automne 2013, mis en ligne le 20 janvier 2014, consulté le 22 avril 2022. URL : http://

journals.openedition.org/medievales/7075; DOI : https://doi.org/10.4000/medievales.7075

Tous droits réservés 
Médiévales 65, automne 2013,p. 19-32

Janet NELSON

\section{DU COUPLE ET DES COUPLES À L'ÉPOQUE CAROLINGIENNE}

Les historiens - mais ils ne sont pas les seuls, car c'est le cas de la plupart des gens - considèrent a priori le couple comme une Bonne Chose, à l'instar de l'amour romantique : il constituerait un véritable marqueur de progrès dans la marche vers la civilisation. Or les hauts médiévistes d'aujourd'hui, particulièrement en France, ont affirmé que le couple avait été inventé à l'époque carolingienne. Et les Français et les chercheurs francophones en général ont par ailleurs tendance à privilégier les structures; les Britanniques en revanche sont prudemment empiriques. Tout en saluant la complémentarité de ces approches ${ }^{1}$, en tant que Britannique, j'aborderai naturellement les choses sous un angle empirique. Je traiterai donc en premier lieu de quelques points méthodologiques et chronologiques, avant d'envisager quelques cas particuliers.

\section{Polyptyques, lois, sources liturgiques : une mutation documentaire}

À partir du viII ${ }^{e}$ siècle, l'accroissement quantitatif et qualitatif de la documentation peut donner une impression de nouveauté dans ce que nous connaissons

1. Je tiens tout d'abord à adresser des remerciements chaleureux à Régine Le Jan, la femme sine qua non de ce dossier sur le couple altomédiéval, puisque ses travaux ont été pionniers en France pour l'étude de la famille et du couple au haut Moyen Âge. Je voudrais ensuite remercier Emmanuelle Santinelli et Sylvie Joye, sine quibus non puisque non seulement elles ont organisé l'atelier qui a débouché sur ce dossier, mais elles ont déjà cultivé ce champ de façon fructueuse. Voir surtout R. LE $\mathrm{J}_{\mathrm{AN}}$, Famille et pouvoir dans le monde franc ( $\mathrm{VII}^{e}-\mathrm{X}^{e}$ siècle), Paris, 1995 ; E. SANTINELLI, Des femmes éplorées? Les veuves dans la société aristocratique du haut Moyen Âge, Villeneuve-d'Ascq, 2003 ; S. JoYe, La Femme ravie. Le mariage par rapt dans les sociétés occidentales du haut Moyen Âge, Turnhout, 2012. Je remercie de tout cœur Sylvie Joye pour la traduction de mon article. 
des couples. Aux $\mathrm{VI}^{\mathrm{e}}$ et $\mathrm{VII}^{\mathrm{e}}$ siècles, ces informations viennent en effet de façon écrasante de sources normatives ou narratives, mais aussi de l'archéologie ${ }^{2}$, alors que pour les $\mathrm{VIII}^{\mathrm{e}}$ et $\mathrm{IX}^{\mathrm{e}}$ siècles, si les sources écrites de ce type subsistent, s'ajoutent à celles-ci les chartes, qui les submergent par leur nombre: quelque 4500 actes sont conservés pour le seul règne de Charlemagne. Dans ces chartes, on voit intervenir essentiellement des laïques - souvent des couples - qui fondent des églises, leur font des donations, ou défendent leurs droits et font mettre par écrit ces actes afin de les conserver dans leurs archives ${ }^{3}$. Cette mutation documentaire n'est pas une coïncidence mais, comme c'est le cas pour d'autres mutations du même genre, l'impression de nouveauté peut être trompeuse du fait que les éléments que nous comparons ne sont pas comparables ${ }^{4}$.

Prenons l'exemple des polyptyques: c'est pendant le règne de Charlemagne et les règnes suivants, mais pas avant, soit au cours du $\mathrm{IX}^{\mathrm{e}}$ siècle, que ces enquêtes ont commencé à recenser littéralement des milliers de couples et leurs enfants. Ceux qui ont constitué ces archives étaient de grands propriétaires ecclésiastiques qui souhaitaient calculer les revenus qui leur étaient dus, en travail ou en argent, et en garder une trace sous une forme plus ou moins standardisée. Le polyptyque était une réponse rationnelle au besoin d'évaluer des ressources économiques, d'assurer la bonne organisation de la main-d'œuvre et d'administrer des domaines à grande échelle ${ }^{5}$. Il s'agissait, dans l'esprit du rédacteur, de l'affirmation d'un pouvoir seigneurial doté d'objectifs politiques et idéologiques à court terme. Les rédacteurs des polyptyques étaient des clercs, et l'organisation du travail qu'ils imposaient était censée répondre aux «lois de la

2. S. F. Wemple, Women in Frankish Society. Marriage and the Cloister, 500-900, Philadelphie, 1981 ; J.-A. McNamara et S. F. Wemple, «Marriage and Divorce in the Frankish Kingdom», dans S. M. Stuard éd., Women in Medieval Society, Philadelphie, 1976, p. 95-124; V. Garver, Women and Aristocratic Culture in the Carolingian World, Ithaca/Londres, 2009; R. STONE, Morality and Masculinity in the Carolingian Empire, Cambridge, 2011, part. p. 247-310 ; J. L. Nelson et A. Rio, «Women in Law Codes», dans J. M. BennetT et R. M. KarRas éd., Oxford Handbook of Women and Gender in the Middle Ages, Oxford, 2013, à paraître; G. Halsall, «Burial Writes: Graves, "Textes" and Time in Early Merovingian Northern Gaul», dans J. JaRnut et M. Wemhoff éd., Erinnerungskultur im Bestattungsritual. Archäologisch-historisches Forum, Munich, 2003, p. 61-74, réimpr. dans G. Halsall, Cemeteries and Society in Merovingian Gaul, Leyde, 2010, p. 214-231.

3. Le chiffre de 4500 est celui de l'état actuel du corpus étudié dans le cadre du projet de base de données «The Making of Charlemagne's Europe», 2012-2015, dirigé par A. Rio, avec la collaboration de J. BRADLEY et al., au King's College de Londres.

4. D. Barthélemy, La Société dans le comté de Vendôme de l'an mil au XIVe siècle, Paris, 1993, p. 19-127 («Les mutations documentaires »); P. STAFFORD, «"La mutation familiale”. A Suitable Case for Caution», dans J. Hill et M. Swan éd., The Community, the Family and the Saint, Turnhout, 1998, p. 103-125.

5. J.-P. Devroey, Puissants et misérables. Système social et monde paysan dans l'Europe des Francs (VI $-I^{e}$ siècle), Bruxelles, 2006, p. 22, 288-292 et 429-441. 
nature ${ }^{6} \gg$. Un polyptyque peut ainsi être comparé au plan de Saint-Gall ou à une idée platonicienne. Et pourtant, dans le même temps, un polyptyque peut laisser voir à la fois des changements récents et les stratégies des couples paysans ${ }^{7}$.

Une des premières et des meilleures historiennes des femmes, Eileen Power, a lu le Polyptyque de Saint-Germain avec le regard d'une spécialiste des sciences sociales, comme si ces données avaient été celles d'un recensement moderne, une description précise de l'organisation des domaines possédés par le monastère éponyme de la région parisienne vers 820. Dans Medieval People (paru en 1924), Eileen Power est partie de quelques lignes à propos de Bodon et Ermentrude, un couple franc présent dans le polyptyque d'Irminon, et a tenté de reconstituer un ensemble de scènes de la vie rurale ${ }^{8}$ :

Bodon doit labourer deux jours par semaine la ferme du domaine du seigneur. Aujourd'hui, Bodon emmène avec lui son gros bœuf et l'aîné de ses trois enfants, le petit Gui, afin qu'il coure à côté du bœuf avec un aiguillon. [...] Pendant ce temps, Ermentrude s'affaire également, car aujourd'hui est due la redevance en volaille: un poulet gras et cinq œufs. Elle laisse son second fils, Gerbert, s'occuper d'Hildegarde, le bébé, pendant qu'elle emmène la redevance en volaille vers la grande maison et la remet à l'intendant. Ensuite, elle se dirige rapidement vers les salles où travaillent les femmes, où elle récupère du lin et de la laine pour en faire des vêtements chez elle. [...] Et dès que le soleil se couche, ils vont au lit.

Les prohibitions touchant le travail dominical sont fortement genrées - et les polyptyques témoignent de cette division. Il est bien connu que Charlemagne aurait dit à son intendant de ne pas laisser les paysans perdre leur temps aux foires ${ }^{9}$. Eileen Power imaginait alors Bodon, Ermentrude et leurs enfants passant une journée à la grande foire de Saint-Denis :

6. J.-P. Devroey, «Femmes au miroir des polyptyques: une approche des rapports du couple dans l'exploitation rurale dépendante entre Seine et Rhin au IX ${ }^{e}$ siècle», dans S. LEBECQ et al. éd., Femmes et pouvoirs des femmes à Byzance et en Occident ( $V I^{e}-\mathrm{XI} I^{e}$ siècle), Villeneuve-d'Ascq, 1999, p. 227-250.; ID., «Men and Women in Early Medieval Serfdom: the Ninth-Century North Frankish Evidence», Past \& Present, 166 (2000), p. 3-30; ID., Puissants..., p. 377-406.

7. É. Renard, «La gestion des domaines d'abbaye aux VIII $-\mathrm{X}^{\mathrm{e}}$ siècles. Notions de base et conseils pour une meilleure compréhension des sources écrites », De la Meuse à l'Ardenne, 29 (1999), p. 115-150; R. FAITH, «Farms and Families in Ninth-Century Provence», Early Medieval Europe, 18 (2010), p. 175-201.

8. Pour cette citation et la suivante, voir E. Power, Medieval People, Reading, 1924, p. 16-19, 24-25 et 31-33; la traduction de ces deux extraits diffère légèrement de celle que l'on trouvera dans EAD., Gens du Moyen Âge. Six croquis de la vie ordinaire, trad. fr. P.-L. VAN BERG, Bruxelles, 2012, p. 55-58 et 73-76.

9. Capitulaire De villis, cap. 54 (éd. A. Boretıus, MGH Capit. Reg. Franc. I, n 32, Hanovre, 1883, p. 88). 
Bodon et Ermentrude ne considéraient pas cela comme une perte de temps. Ils se disaient qu'ils voulaient acheter du sel afin de saler leur viande pour l'hiver, ou du vermillon pour teindre une robe pour le bébé. [...] Et il y avait toujours des jongleurs, des joueurs de tambourin, des montreurs d'ours et des ménestrels pour faire disparaître les quelques sous qui se trouvaient dans la poche de Bodon. C'était une famille très fatiguée mais très heureuse qui montait dans son chariot pour rentrer se coucher. [...] Cela vaut vraiment la peine de passer quelques moments avec [eux]. L'Histoire est largement faite de Bodons.

Elle aurait dû ajouter : « et d'Ermentrudes ». Mis à part cela, Eileen Power a assez bien réussi à donner un aperçu de la vie réelle à partir d'un extrait de polyptyque, et à rendre accessible à un large public la vie de couple au haut Moyen Âge ${ }^{10}$.

Mais - car il y a souvent un mais - nous pouvons échouer dans nos efforts à reconstituer la vie réelle, et nos tentatives pour rendre les choses accessibles peuvent aussi nous amener à distordre la réalité. Il y a des groupes familiaux qui ne se forment pas autour d'un couple. S'il est vrai qu'il y a un couple au centre de la plupart des familles, cela ne signifie pas que la relation soit fondée sur un principe d'égalité. Les informations présentes dans les polyptyques renvoient souvent à l'inégalité des partenaires : ainsi les cas d'hypergamie, où des hommes épousent des femmes d'un statut plus élevé (un servus épousant une colona), sont de loin plus nombreux que ceux où des ancillae épousent des coloni; et l'idée même qu'une union suppose un choix mutuel ignore non seulement les projets des parents et des membres plus âgés de la famille, mais aussi les intérêts seigneuriaux, les redevances dues, qui demeurent cachés derrière la description des registres. Est-ce que le «couple» renvoie à quelque chose comme un «compagnonnage du mariage» (companionate marriage), dont les spécialistes du début de l'époque moderne croient qu'il est une spécificité de leur période, tout comme les spécialistes du Moyen Âge central l'imaginent, eux, apparaître à leur période? C'est ce que démontrerait 1' «humanité » du comte Geoffroy Martel d'Anjou et de sa femme qui, siégeant comme d'autres couples comtaux

[...] à la plus haute fenêtre de leur château nouvellement construit, [ont vu] une météorite tomber plus bas, dans le jardin et, courant jusqu'à l'endroit où celle-ci était tombée, [ont fait le vœu] de fonder une abbaye dédiée à la Sainte Trinité

10. M. Berg, A Woman in History: Eileen Power, Cambridge, 1996. J'ai l'impression que c'est par Eileen Power que, longtemps après sa mort en 1940, Bodon a fait son entrée dans le discours des sciences humaines aux États-Unis: voir par exemple l'économiste Axel LEIJONHUFVUD, «The Individual, the Market and the Industrial Division of Labour», 1986, réimpr. dans C. MoNGARDIN éd., L'Individuo e il mercato, Rome, 1995, p. 61-78, et le sociologue R. HARDIN, «From Bodo Ethics to Distributive Justice», Ethical Theory and Moral Practice, 2 (1999), p. 399-413 (p. 401-402). 
en mémoire des trois fragments scintillants qui avaient brillé devant leurs yeux émerveillés ${ }^{11}$.

Cet épisode, fondé sur une histoire de miracle, peut-il être considéré comme un moment représentatif de la «vie de couple» ? Le vœu était-il une idée de Geoffroy ou de son épouse? La pénurie d'exemples similaires avant le $\mathrm{XI}^{\mathrm{e}}$ siècle constituet-elle la preuve d'une absence ou une absence de preuve?

Les textes normatifs sont moins évocateurs que les récits de miracles ou même les polyptyques, mais ils permettent d'effectuer une comparaison sur la longue durée. Bien que les périodes les plus sombres du $\mathrm{xx}^{\mathrm{e}}$ siècle aient rendu délicat l'usage du terme de collaboration, la conlaboratio du haut Moyen Âge semble avoir évoqué d'une manière positive ce qu'il signifie étymologiquement: l'exploitation du sol par des époux travaillant ensemble (et ici le terme «travail» renvoie également à la gestion). Un formulaire franc copié au milieu du $\mathrm{IX}^{\mathrm{e}}$ siècle, mais fondé sur un texte beaucoup plus ancien, évoque ainsi un échange de dons entre des époux dépourvus d'enfants: «tous leurs biens meubles et leur conlaboratio $[. .$.$] de telle sorte que chaque partie dote son égale de sa part { }^{12} \gg$. On observe un glissement de la notion de «deux parties» (partes) vers celle de « deux pairs » (pares), ou égaux. Cette terminologie n'était pas nouvelle. Au milieu du $\mathrm{VII}^{\mathrm{e}}$ siècle, la Loi des Ripuaires prévoyait que la femme qui survivait à son mari devait recevoir 55 sous et pouvait réclamer un tiers de tous les biens sur lesquels ils avaient travaillé ensemble (simul conlaboraverint). Louis le Pieux, en 821, ordonna que les missi s'assurent que la veuve du titulaire d'un bénéfice reçoive la tertiam partem conlaborationis quam simul in beneficio conlaboraverunt ${ }^{13}$. On voit qu'ici la collaboration du couple ne cesse pas avec la mort d'un des époux. On peut aussi remarquer qu'un nombre relativement élevé de femmes sont mentionnées dans les documents qui enregistrent des conflits judiciaires. Ainsi, un procès local référé à un tribunal constitué soit par l'empereur soit (possibilité intéressante) par des hommes du voisinage - des hommes qui connaissent bien les lois - a rendu un jugement décisif à propos de la prééminence sur la Lex Salica de la consuetudo qui préconise de se rendre à l'avis des parents dans un

11. R. W. Southern, The Making of the Middle Ages, Londres, 1953, p. 91, faisant référence à L. HalPhen, Le Comté d'Anjou au XI 'e siècle, Paris, 1906, p. 351-352.

12. Formulae Salicae Lindenbrogianae $\mathrm{n}^{\circ} 20$ (éd. K. Zeumer, Formulae Merowingici et Karolini aevi, MGH Leges V, Hanovre, 1886, p. 281) : «et omne praesidium vel conlaboratio eorum inter se pars parti visi sunt condonari in tali vero tenore ut quisque de eis pari suo in hoc seculo superstes fuerit omnes res quas dedit et quas accepit in suo iure vel dominatione recipere debeat...».

13. Lex Ribuaria cap. 41, 2 (éd. F. Beyerle, MGH Leges III-2, Hanovre, 1954, p. 95); Capitulare missorum, cap. 9 (éd. A. Boretıus, MGH Capit. I, nº 148, p. 301). 
cas de formation d'un couple par remariage d'une veuve: aperçu exceptionnel de la pratique juridique aux temps carolingiens ${ }^{14}$.

L'intérêt de l'Église pour les rites de passage les plus importants pourrait laisser penser que la liturgie altomédiévale donnait beaucoup d'importance au couple. En réalité, les plus anciennes bénédictions nuptiales sont liées au voile posé sur la tête de la sponsa, l'épouse, et, bien qu'on y trouve les termes foedus, societas et consortium, les textes se concentrent sur la figure de la mariée ${ }^{15}$.

Dans un sacramentaire gélasien du VIII ${ }^{\mathrm{e}}$ siècle, le soi-disant Sacramentaire de Gellone, la prière «Père, créateur du monde» se poursuit ainsi :

Fais que la femme fidèle et chaste se marie dans le Christ, et demeure l'imitateur féminin des saintes femmes. Fais qu'elle soit aussi aimable que Rachel l'était pour son époux et aussi sage que Rébecca, et que sa vie soit aussi longue et fidèle que celle de Sarah. Que l'auteur des mensonges n'obtienne rien d'elle par ses actions. Qu'elle demeure vouée à la foi et à ce qui est commandé aux femmes, en servant Dieu avec dévotion. Qu'elle renforce sa faiblesse par la force de la discipline et, en rejoignant le lit d'un homme, qu'elle fuie les contacts illégitimes de la vie ${ }^{16}$.

Ce même sacramentaire contient onze prières de messe pour la femme stérile, dont la deuxième dit:

Dieu, toi qui as daigné écouter la prière de ton serviteur Isaac pour sa femme et lui accorder la possibilité de concevoir, entends les prières de ta servante et fais qu'elle puisse concevoir un enfant, afin que le bien-fondé de l'espoir qu'elle a mis en ta clémence puisse être confirmé quand elle recevra le don qu'elle a demandé ${ }^{17}$.

En effet, ce n'est pas avant le tournant du $\mathrm{IX}^{\mathrm{e}}$ et du $\mathrm{X}^{\mathrm{e}}$ siècle que les sacramentaires proposent des prières pour le couple ${ }^{18}$.

Les invocations pour être purifiée et fortifiée par Dieu dans les prières carolingiennes destinées aux sponsae renvoient aux préoccupations continuelles

14. Capitula legi salicae addita, cap. 8 (éd. A. Boretıus, MGH Capit. I, nº 142, p. 293). Voir J. L. Nelson, «The Carolingian Renaissance Revisited», à paraitre; T. FAulkner, The Frankish Leges in the Carolingian Period, thèse de doctorat, université de Cambridge, 2009, p. 148.

15. J. ÉveNOU, «Le mariage», dans A.-G. MARTIMORT éd., L'Église en prière. Introduction à la liturgie, vol. III, Les Sacrements, Paris, 1984, p. 201-224, avec des citations d'anciens sacramentaires (Veronense $\mathrm{n}^{\circ} 1110$, Hadrianum $\mathrm{n}^{\circ} 837,838 \mathrm{a}$; Gelasianum $\mathrm{n}^{\circ}$ 1449, 1451, 1453); P. L. Reynolds, Marriage in the Western Church. The Christianization of Marriage during the Patristic and Early Medieval Periods, Leyde, 1994 (Vigiliae Christianae, Suppléments, vol. 24), p. 381-385.

16. Liber Sacramentorum Gellonensis, dit Sacramentaire de Gellone, $\mathrm{n}^{\circ} 2636$ (éd. A. Dumas et J. Deshusses, Corpus Christianorum, Series Latina, CLIX, Turnhout, 1981, p. 412-413).

17. Sacramentaire de Gellone, $\mathrm{n}^{\circ}$ 2646-56, p. 415-417.

18. Oratio pro coniugandis, dans The Leofric Missal, nº 2439 (éd. N. OrChaRd, Henry Bradshaw Society, CXIII, Londres, 2002, vol. II, p. 424); Actio nuptialis, dans Sacramentarium Fuldense, no 2605-2617 (The Sacramentary of Fulda, éd. G. Richter et G. SCHÖNFELDER, Fulda, 1912, réimpr. Henry Bradshaw Society, CI, Londres, 1977, p. 323-326). 
du clergé à propos de la pollution infligée par la femme à l'espace ecclésial la pollution étant d'autant plus grave qu'on s'approche davantage de l'autel. Rappelons-nous que la mariée était un élément extérieur pénétrant dans une maison conjugale qui était celle de son époux. Il n'existe aucun texte carolingien comparable à l'Histoire des comtes de Guînes rédigée vers 1200 par le prêtre Lambert d'Ardres, texte auquel Georges Duby a encore ajouté du piment par une réécriture assez leste. Lambert et ses deux fils, également prêtres, y font le tour du lit dans lequel les jeunes mariés sont couchés, en aspergeant ceux-ci d'eau bénite: «en ce moment nocturne, [...] ils encensent le lit, ils le sacralisent, ils en font une sorte d'autel ${ }^{19}{ }^{»}$. Il n'existe aucune preuve que de telles cérémonies aient existé à l'époque carolingienne. Cependant, on peut deviner des préoccupations et des anxiétés similaires derrière les fonctions apotropaïques et d'exorcisme des prières que je viens de citer. Un synode réuni par le roi Pépin en 755 décrète que les mariages de tous les laïques, nobles et non-nobles, doivent être conclus en public, mais rien n'est dit d'éventuelles bénédictions ${ }^{20}$. Jonas d'Orléans conseillait aux laïques de faire bénir leur mariage par un prêtre pour protéger les droits à l'héritage de leur descendance ${ }^{21}$. Cette injonction est cependant isolée: rien n'est dit à ce sujet dans les statuts épiscopaux du IX $\mathrm{I}^{\mathrm{e}}$ siècle; et Dhuoda, qui écrit en 842, mentionne qu'elle a été mariée le 29 juin 824 au palais d'Aix-laChapelle, mais elle ne dit rien d'une église ni de la participation de prêtres. J'en conclus que la prise en charge religieuse du couple par la liturgisation des rites du mariage avait alors fait peu de progrès dans le monde carolingien. Bien que les ecclésiastiques aient élaboré des règles à propos de l'inceste, la mainmise sur le mariage restait aux laïques, spécialement aux époux qui étaient, ou devaient devenir, aussi des pères.

\section{Trois couples atypiques}

Trois cas plutôt étranges, mais en même temps représentatifs, vont illustrer ces points. Un premier couple atypique est celui formé par Berthe et Angilbert. Berthe était la deuxième fille de Charlemagne. Un poète contemporain, peutêtre Angilbert lui-même, décrit ainsi ses frères et sa sœur aînée, puis Berthe ellemême sortant d'Aix en procession pour se rendre à la chasse en 799:

19. G. DuBy, Le Chevalier, la femme et le prêtre. Le mariage dans la France féodale, Paris, 1981, p. 275.

20. Concile de Ver (755), cap. 15 (éd. A. Boretius, MGH Capit. I, nº 14, p. 36).

21. Jonas D'OrléAns, De institutione laicali 2, 2 (PL 106, col. 171). Voir K. HeEnE, The Legacy of Paradise. Marriage, Motherhood and Women in Carolingian Edifying Literature, Francfort, 1997, p. 69, n. 44. 
Elle brille en compagnie de nombreuses autres jeunes femmes, virile par sa voix et par son esprit, resplendissante dans son vêtement et dans son visage, avec un visage, une bouche, une allure et des yeux qui rappellent les traits de son père ${ }^{22}$.

Charlemagne était en effet un père atypique: il ne voulait pas laisser ses filles se marier, disait Éginhard, parce qu'il ne pouvait pas vivre sans leur contubernium - leur compagnie ${ }^{23}$. Mais le terme choisi par Éginhard est étrange lui aussi, car il renvoie littéralement, soit au partage d'une tente, ce qui suppose un compagnonnage guerrier plutôt mâle, soit au concubinage, une relation hétérosexuelle sans mariage ${ }^{24}$. En réalité, celui qui partageait la tente de Berthe n'était pas son père mais Angilbert, l'un des principaux personnages de l'entourage de son père, un jeune aristocrate franc, poète de cour, agent de confiance envoyé loin de la cour pour des missions au service du roi, à qui était échue la riche abbaye de Saint-Riquier ${ }^{25}$. La cour était le foyer de cet amoureux installé, mais il est probable qu'il résida à mi-temps à Saint-Riquier pour y exercer la charge d'abbé jusqu'à sa mort le 18 février 814. La suite est racontée par l'historien Nithard: après avoir annoncé à ses lecteurs que le corps d'Angilbert avait été déterré à Saint-Riquier vingt-huit ans après sa mort et avait été trouvé intact - ce qui signifiait, en d'autres termes, qu'il était un saint -, Nithard continue en décrivant l'illustre famille d'Angilbert, qui était tenue en grande estime par Charlemagne. Il ajoute alors, en un coup de théâtre magistral: «d'une fille de ce grand roi, dont le nom était Berthe, il [Angilbert] engendra mon frère Hartnid et moi-même, Nithard... Voilà donc par ailleurs ce qu'il en est de mes origines familiales... ${ }^{26} »$. De telles liaisons étaient plus difficiles à accepter selon les conceptions des années 840. Pourtant, Berthe et Angilbert avaient cohabité à la cour sans vergogne dans les années $790^{27}$. Charlemagne, ce pilier de l'ordre

22. J. L. Nelson, «La cour impériale de Charlemagne», dans R. Le JAN éd., La Royauté et les élites dans l'Europe carolingienne, Villeneuve-d'Ascq, 1998, p. 177-191 (p. 186-188).

23. Éginhard, Vita Karoli Magni, cap. 19 (éd. O. Holder-Egger, MGH SRG 25, Hanovre, 1911, p. 25).

24. J. L. Nelson, «Women at the Court of Charlemagne», dans J. C. Parsons éd., Medieval Queenship, New York, 1993, p. 43-61.

25. J. L. Nelson, «Charlemagne - pater optimus?», dans P. Godman, J. Jarnut et P. JohaneK éd., Am Vorabend der Kaiserkrönung, Stuttgart, 2002, p. 269-281 (p. 270). On peut se demander si, dans le cas d'Angilbert, le terme «abbé laïque» est bien le mot juste.

26. Nithard, Histoire des fils de Louis le Pieux, IV, 5 (éd. et trad. P. Lauer, revue par S. GLANSDORFF, Paris, 2012, p. 150).

27. H. Fichtenau, Das Karolingische Imperium, Zürich, 1949, p. 106-107, citant Alcuin, Epistulae, 97 (éd. E. DümmLER, MGH Ep. IV, Berlin, 1895, p. 141), pensait qu'Alcuin savait très bien qu'Angilbert avait, comme lui-même, entretenu des relations sexuelles défendues, ce qui expliquerait l'explosion de nombrilisme, de sentimentalité et de panique eschatologique présente dans les lettres du vieil homme vers la fin des années 790. Voir le commentaire bien nuancé de S. A. RABe, Art and Politics at Saint-Riquier. The Symbolic Vision of Angilbert, Philadelphie, 1995, 
patriarcal, avait voulu l'existence du couple, et aussi, sans doute, sa fin. Son petit-fils considérait ce passé avec gratitude.

Un second couple atypique est celui formé par Dhuoda et Bernard. Le Manuel de Dhuoda destiné à son fils Guillaume fournit beaucoup d'indications sur sa relation avec son époux - son dominus et senior - mais il dissimule également beaucoup de choses. Selon Pierre Riché, Dhuoda prêchait une religion de la paternité ; s'il en allait ainsi, cette divinité incarnée avait des pieds d'argile ${ }^{28}$. Dans les années 828-833, Dhuoda, quel que soit l'endroit où elle vivait alors, devait avoir eu vent des rumeurs qui circulaient à la cour et au-delà au sujet de la liaison entre Bernard et l'impératrice Judith ${ }^{29}$. Écrivant une décennie plus tard, lors d'un autre moment de crise, Dhuoda demandait à Guillaume de «prier pour son père pendant la durée de sa vie de façon constante et assidue, et de pousser le clergé à faire de même, de telle sorte que Dieu lui accorde paix et concorde avec tous », ajoutait-elle, «si cela était possible»; et elle qualifiait ainsi ses espoirs concernant la vie éternelle de Bernard «si Dieu le veut, en prêtant attention à ce qu'il ait accompli dans cette vie une pénitence fructueuse et pratiqué l'aumône généreusement $»^{30}$. Dhuoda demandait également à son fils de «prier pour les parents décédés de [son] père ». Régine Le Jan a assurément raison de souligner ici la référence à la lignée paternelle et, au-delà, de relier très concrètement le devoir de prier aux biens hérités de la branche paternelle - et donc de lier la memoria avec l'hereditas et les hereditates ${ }^{31}$ :

Prie de toutes tes forces pour l'âme de ceux qui possédaient tout [ce que ton père possède désormais]. Lui-même ne peut prier maintenant car il est occupé par de nombreux problèmes. Mais toi, tu peux et tu dois prier pour eux.

Dhuoda ajoute plus loin que Guillaume doit partager ce devoir de prière avec son petit frère. Ce que le père était trop occupé pour faire, ses fils se devaient de

p. 73, qui caractérise néanmoins (à tort) la relation entre Angilbert et Berthe comme Friedelehe: voir à ce sujet R. M. KARRAS, «The History of Marriage and the Myth of Friedelehe», Early Medieval Europe, 14 (2006), p. 119-151 (p. 150).

28. Dhuoda, Manuel pour mon fils (éd. et trad. P. Riché, Paris, 1975, Introduction, p. 27). Voir J. L. Nelson, «Dhuoda», dans P. Wormald et J. L. Nelson éd., Lay Intellectuals in the Carolingian World, Cambridge, 2007, p. 106-120.

29. Annales Mettensis priores, s. a. 830 (éd. B. von Simson, MGH SRG 10, Hanovre, 1905, p. 97); Agobard, Liber Apologeticus I, cap. 2 (éd. L. VAN AcKer, Agobardi Lugdunensis Opera omnia, Corpus Christianorum Continuatio Medievalis, LII, Turnhout, 1981, p. 309); TheGAN, Gesta Hludowici imperatoris, cap. 36 (éd. E. TREMP, MGH SRG 64, Hanovre, 1995, p. 222); Annales Bertiniani, s. a. 830 et 831 (éd F. Grat et al., Paris, 1964, p. 2-5).

30. Dhuoda, Manuel..., op. cit., VIII, 7, p. 310.

31. R. LE JAN, «Dhuoda ou l'opportunité du discours féminin», dans C. LA RocCA éd., Agire da Donna, Turnhout, 2007, p. 109-128 (p. 114-120). 
l'accomplir ${ }^{32}$. Il y a de l'amertume, et même de l'ironie, dans cette affirmation extraordinaire.

Le témoignage de Dhuoda elle-même ne permet pas de savoir combien de temps ce couple a passé ensemble durant sa vie maritale, que ce soit à Aix ou en Septimanie. L'espace de quatorze années qui sépare la naissance de leurs deux fils (29 novembre 826/22 mars 841) ne suggère pas que ces parents aient joui d'une proximité physique intense ou continuelle. Dhuoda vivait à Uzès, sub iussione senioris mei - «selon le commandement de mon seigneur ${ }^{33}$ ». La description qu'elle fait de son servitium et du soin porté aux utilitates de son mari (ses intérêts) en Septimanie, ainsi que de son attention à se débarrasser des dettes non seulement envers les chrétiens, mais aussi envers les juifs, suscite la pitié; on ressent plus encore sa douleur lorsqu'elle annonce que Bernard emporte avec lui son plus jeune fils, dont elle ne connaît pas encore le nom. Bernard, tiraillé entre la loyauté due à deux rois carolingiens qui s'opposent, a utilisé ses deux fils en recommandant chacun à un souverain différent: l'un immédiatement, et l'autre de façon anticipée. Ce mari est allé très loin dans les contraintes qu'il a imposées à une parentalité partagée. Aussi peut-on dire que ce couple n'était pas seulement étrange mais aussi malheureux, et même tragique.

Le troisième couple atypique que j'évoquerai est celui formé par Northilde et Agambert. Northilde était une noble franque incapable de supporter plus longtemps les inhonesta (les actes déshonorants) que lui infligeait son mari Agambert, convoqué à comparaître en public face à Louis le Pieux à la grande assemblée d'Attigny de 822 afin que le divorce soit accordé à Northilde ${ }^{34}$. La prééminence de la monogamie n'était pas encore alors assurée. Hincmar de Reims est le seul à rapporter dans son traité De divortio de 860 un compte-rendu de l'affaire, fondé sur ses souvenirs personnels et peut-être sur quelques notes. Il la considérait comme un cas exemplaire des «limites posées par nos ancêtres [...] qui ne doivent pas être transgressées ». Voici ce qui arriva:

L'empereur envoya [Northilde] se présenter au synode afin que l'autorité des évêques (episcopalis auctoritas) décide de ce qui devait être fait. Mais l'ensemble des évêques la renvoya au jugement des laïques et des hommes mariés ( $a d$ laicorum ac coniugatorum eam remisit iudicium), afin qu'ils puissent rendre un jugement entre elle et son mari, car ces laïques connaissaient ce genre de choses et possédaient une connaissance très fine des lois de ce monde (qui de talibus negotiis erant cogniti et legibus saeculi sufficientissime praediti) et alors [ils dirent] que cette femme devrait se soumettre au jugement rendu par ces hommes, et elle devrait respecter ce qu'ils auraient décidé concernant son accusation (quod de

32. Dhuoda, Manuel..., op. cit., VIII, 14, p. 318-321.

33. Ibid., préface, p. 86-87.

34. Ce n'est pas totalement explicite, mais cette lecture semble très vraisemblable. 
quaestione sua decrevissent, sine repetitione teneret), sans aucun [droit d']appel. [...] Cette décision des évêques (sacerdotalis discretio) plut aux nobles laïques, parce qu'un procès concernant leurs épouses ne leur avait pas été retiré, et parce qu'aucun tort n'avait été fait aux lois civiles par le pouvoir épiscopal (nobilibus laicis sacerdotalis discretio placuit, quia de suis coniugibus eis non tollebatur iudicium nec a sacerdotali ordine inferebatur legibus civilibus praeiudicium). Alors ils [les nobles laïques] proclamèrent la loi en réponse à la requête de la femme, et ils mirent un terme à sa demande par un jugement légal ${ }^{35}$.

Des éléments de haute politique ont joué un rôle dans ce scénario. Ont également joué un rôle les manifestations précoces de la lecture des Écritures par les laïques, ainsi que les avancées qui s'ensuivirent dans le domaine de la discipline pénitentielle, en particulier à propos des affaires d'inceste (la prohibition de l'inceste, habilement instrumentalisée, pouvaient permettre le divorce) et des affaires de rapt $^{36}$. Certaines circonstances, telles que l'exil forcé, pouvaient permettre de s'adonner à la monogamie en série; le mariage honorable, associé à un douaire et impliquant des partenaires de statut social plus ou moins égal, était de plus en plus souvent nettement différencié du concubinage entre un libre ou un noble et une ancilla. Ruth Karras a remisé à juste titre la Friedelehe aux oubliettes ${ }^{37}$. Il est vrai que les raisons qui pouvaient justifier un divorce favorisaient en majorité les maris - ainsi la fornication de l'épouse donnait à son mari le droit de divorcer, alors que l'inverse n'était pas vrai, ce qui montre clairement qu'un double standard était à l'œuvre. De même, certains époux considéraient apparemment qu'ils étaient dans leur droit en divorçant d'une épouse perpétuellement malade ${ }^{38}$. Cependant, la loi canonique et les lois civiles condamnaient la violence masculine, d'autant que la cruauté exercée envers une femme amoindrissait l'honneur de sa famille. Et des croyances culturelles fortes soutenaient que la virilité vertueuse impliquait de protéger les faibles - dont les femmes représentaient une figure essentielle. Par ailleurs, les formulaires

35. HinCMAR, De divortio Lotharii regis et Theutbergae reginae, Responsio 5 (éd. L. BöHRINGER, MGH Conc. IV, Supplementum, Hanovre, 1992, p. 141-142). Voir J. L. Nelson, «Hunnish Scenes/ Frankish Scenes: A History that Stands Still?», dans J. L. Nelson, S. Reynolds et S. Johns éd., Gender and Historiography. Studies in the Earlier Middle Ages in Honour of Pauline Stafford, Londres, 2012, p. 175-190.

36. À ce propos et pour ce qui suit, se reporter désormais à R. STONE, «Bound from Either Side: The Limits of Power in Carolingian Marriage Disputes, 840-870 », Gender and History, 19 (2007), p. 467-482; EAD., «The Invention of a Theology of Abduction: Hincmar of Reims on raptus », Journal of Ecclesiastical History, 60 (2009), p. 433-448; EAD., Morality and Masculinity in the Carolingian Empire, Cambridge, 2012, surtout p. 33-35, 249-255, 255-267 et 292-301; E. SANTinelli éd., Répudiation, divorce, séparation dans l'Occident médiéval, Valenciennes, 2007; S. Joye, La Femme ravie..., surtout p. 355-434 et 443-448.

37. R. KarRas, «The History of Marriage...», p. 119-151.

38. JonAS D'OrLÉANS, De institutione laicali, II, 12, col. 190-191. 
continuaient à être copiés aux $\mathrm{VIII}^{\mathrm{e}}$ et $\mathrm{IX}^{\mathrm{e}}$ siècles, demandes de divorce par consentement mutuel incluses ${ }^{39}$. Mais en matière d'histoire du couple, ce qui ressort clairement du cas de Northilde, c'est la prédominance et la connivence des «nobles laïques» et du clergé pour maintenir le patriarcat.

\section{Questions carolingiennes}

Les histoires de couple que je viens de retracer furent pour la plupart fort tristes. Je voudrais terminer dans l'esprit d'Eileen Power en évoquant quelques questions/réponses, apparemment composées à la cour de Charlemagne, et conservées dans un manuscrit d'usage courant daté du début du $\mathrm{IX}^{\mathrm{e}}$ siècle. Les sujets abordés évoquent des hommes et des femmes, et ceux qui étaient engagés dans ces discussions pouvaient être des femmes, et peut-être des couples. En même temps, ce type d'ouvrage nous transmet le ton de voix pleines d'humanité et d'humour. Ces séries de questions m'ont été soumises par David Ganz, qui souhaitait «offrir une source d'enseignement pour ceux qui voudraient mieux comprendre l'esprit franc ${ }^{40} »$.

Question: Donc, j'aime une femme?

Réponse: Alors tu aimes la mort. Car, comme il a été dit, «l'homme qui est un amant trop ardent de sa propre femme est un adultère ${ }^{41} \gg$. Mais tout amour destiné à une autre femme [que sa propre épouse] est dégoûtant, qu'il soit grand ou petit.

Question: Donc la femme est la mort?

Réponse: Non, elle n'est pas la mort, mais parce qu'elle est la cause de la mort, on pense que c'est par une femme que nous devenons tous mortels.

Question: Donc la femme doit être haïe?

Réponse: Seulement à cause du péché, mais pas à cause de sa nature. C'est pourquoi tu dois détester le vice, mais aimer la créature. Car cette femme est un être humain et l'œuvre de Dieu, et donc une Bonne Chose.

39. S. F. Wemple, Women..., p. 77; A. Rio, The Formularies of Angers and Marculf. Two Merovingian Legal Handbooks, Liverpool, 2011, p. 97-98.

40. D. GAnZ, «Some Carolingian Questions from Charlemagne's Days», dans P. Fouracre et D. Ganz éd., Frankland. The Franks and the World of the Early Middle Ages. Essays in Honour of Dame Jinty Nelson, Manchester, 2006, p. 90-100 (p. 90 et 93). Voir aussi D. Bullough, «Charlemagne's Court Library Revisited», Early Medieval Europe, 12 (2004), p. 339-363 (p. 358359). Je suis très reconnaissante envers mon ami David Ganz pour ses encouragements généreux à me pencher sur le ms. Paris, BnF, lat. $4629, \mathrm{f}^{\circ} 15 \mathrm{v}^{\circ}-18 \mathrm{v}^{\circ}$.

41. La citation est de JÉrôme, Adversus Iovinianum, I, 49. 
Ces questions sont encore logiques aujourd'hui. Je poserai donc une question finale de mon propre cru: sommes-nous sur la voie de comprendre ce que les Francs pensaient du couple?

Traduction Sylvie Joye.

Janet NeLSON - King's College London, Department of History, Strand, London WC2R 2LS, Grande-Bretagne

\section{Du couple et des couples à l'époque carolingienne}

Le couple a souvent été considéré par les médiévistes occidentaux à la fois comme une donnée de l'organisation d'une société civilisée et comme une invention de la période carolingienne. Eileen Power, une sociologue intéressée par le Moyen Âge, a proposé une vision bucolique du couple tel qu'il apparaît dans les polyptyques. Le présent article propose de nuancer ce tableau. Il réexamine la typologie des groupes familiaux, les unions hyper- et hypogamiques, et la pertinence de la notion de «compagnonnage du mariage». Les sources légales éclairent la collaboratio au sein des couples. Le couple ne vient qu'en second, après la mariée, dans les plus anciens textes liturgiques occidentaux. Il démontre que trois exemples de couples bien connus, souvent vus comme représentatifs, peuvent en réalité être considérés comme curieux à divers égards. Enfin, c'est de la cour de Charlemagne que nous vient un aperçu d'une discussion étonnamment empathique au sujet des relations homme-femme.

mariage - genre - polyptyques - lois - liturgie

\section{Couple and Couples from the Carolingian Times}

The couple has often been taken by western medievalists as a given of civilized social arrangements and an invention of the Carolingian period. Eileen Power, a social scientist with medieval interests, took a rosy view of the couple in the polyptychs. This paper proposes some qualifications. Varieties of family group, hypergamous and hypogamous unions, and the relevance of "companionate" marriage, are re-examined. Legal sources throw light on couples' collaboratio. The couple takes second place to the bride in early western liturgical texts. Three well-known couples sometimes considered representative are shown to be, variously, odd. Finally, from Charlemagne's court comes a glimpse of an unexpectedly sympathetic discussion of man-woman relationships.

marriage - gender - polyptychs - law - liturgy 
